\title{
Six years of MEMO: readers survey 2012
}

\author{
Wolfgang Hilbe
}

Published online: 15 June 2013

(C) Springer-Verlag Wien 2013

It has been 6 years since $M E M O$ was founded; 6 volumes included 35 issues with 380 articles. The Editorial Board comprises of 60 experts coming from 15 different countries with special focus on Central European Nations. More than 400 experts were engaged as reviewers or authors and supported the Editorial office to manage a tremendous work-flow (see Table 1).

Substantial support came from our Section Editors (SE):

- SE Breast Cancer: István Láng, Hungary

- SE Gastrointestinal Cancer: Vladimir M. Moiseyenko, Russia

- SE Hematology: Eberhard Gunsilius, Austria

- SE Neurooncology: Silvia Hofer, Switzerland

- SE Pediatric Oncology: Michael Dworzak, Austria

- SE OeGHO: Günther Gastl and Hellmut Samonigg, Austria

- SE CECOG:

Thomas Brodowicz and Christoph Zielinski, Austria

The concept of MEMO has been to offer a professional review on current research and development in the field of hematology and oncology relevant for daily practice. Therefore, MEMO includes editorials and comments, peer-reviewed original reports, short reviews, case reports and controversies, articles explaining the biology of neoplasia, and congress reports including qualified comments. As a predominant European journal, $M E M O$ aims at highlighting the local peculiarities of various regions and at being a forum for the presentation of ongoing clinical and basic research. In 2007, MEMO became

\section{Prof. Dr. W. Hilbe $(\bowtie)$}

Department for Internal Medicine V, Hematology

and Oncology, Medical University Innsbruck,

Anichstraße 35, 6020 Innsbruck, Austria

e-mail: Wolfgang.Hilbe@i-med.ac.at the official journal of the Central European Cooperative Oncology Group (CECOG) and in 2012 of the Austrian Society of Hematology and Oncology (OeGHO).

\section{Readers' poll}

In 2012 the Springer Company initiated a readers' poll to evaluate the acceptance of MEMO. Overall, 72 (4\%) of 1,750 questionnaires were completed and served as the basis for the actual analysis. The majority of these readers are employed at a hospital. The readership is composed of specialists in internal medicine $(50 \%)$, gynecology (13\%), radiotherapy $(8 \%)$, basic research $(7 \%)$, surgery (6\%), pathology (6\%), and pneumoloy (4\%). For them, a medicinal journal should offer a high quality of information, authors with a high expertise, clear structured presentation of the articles and practical relevance of the contents. These preconditions have been sufficiently fulfilled by MEMO. The most important categories of articles have been short reviews and reviews.

Overall, $85 \%$ of the readers were fine with the selection of the topics. Around $69 \%$ of them used three or four issues per year. As an overall rating, $82 \%$ of the readers appreciated MEMO; only $15 \%$ qualified the journal to

Table 1 2012-One year of MEMO

Four issues including 308 pages

Eighty-two submitted manuscripts, 78 published

Authors come from 20 different countries (Europe, Asia, Africa, America)

Invited reviewers ( $n=454)$ from 20 countries

Qualification of the publications at initial submission by the reviewers: "accept as is" $14 \%$, "major revsions" $19 \%$, "minor revisions" $65 \%$, "reject" $2 \%$

Finally, $9 \%$ of the manuscripts had to be rejected by the responsible Editors

Communications of the Editorial Office: $>4,000$ emails 
be of minor relevance. Some negative aspects have also been forwarded. So, several interviewees asked for more articles covering the fields of radiotherapy, urology, gynecology, case reports, and congress updates. Additionally, geriatric aspects, quality of life, and "state-ofthe-art" topics should be strengthened.

Asked for a rating of properties of $M E M O$, most of the parameters were qualified to be excellent (authors, actuality, information content, structure of the issues, and spectrum of topics), two were qualified to be good (relating to daily practice and internationality).

The results of this poll were extensively discussed at the last Editorial Board Meeting. The well taken points made by our readers will help us to further develop MEMO in the next years. First of all, we try to focus more on the practical relevance of our articles and on the extension of the expertise of other oncological specialties. As a first step, our Board of Section Editors was extended in 2012: István Láng from Hungary now serves as a SE for Breast Cancer, Vladimir M. Moiseyenko from Russia for Gastrointestinal Cancer, and Silvia Hofer from Switzerland for Neurooncology.

As the Editor-in-Chief, I see that our concept proves to be highly accepted, but there is still room for improvement. It is my privilege to thank all those readers, who gave us their feedback, and all the authors and reviewers who made this success possible.

In accordance with a prior editorial (Vol. 1, issue 4) "we keep on giving our best, making $M E M O$ a leading journal in Europe".

With my best regards, yours Wolfgang Hilbe (Editor-in-Chief) 